\title{
11. PRELIMINARY PALEOMAGNETIC RESULTS, LEG 22
}

\author{
Richard D. Jarrard, Scripps Institution of Oceanography, La Jolla, California \\ and \\ John G. Sclater, Massachusetts Institution of Technology, Boston, Massachusetts
}

\section{METHODS}

Paleomagnetic sampling was carried out on Miocene through Upper Cretaceous sediments recovered at Site 212, located at $19^{\circ} 11.3^{\prime} \mathrm{S}, 99^{\circ} 17.8^{\prime} \mathrm{E}$ in the Wharton Basin. The 6 -cc rectangular samples were taken in apparently undisturbed, firm to very firm zeolitic clays. Samples were kept sealed and refrigerated, except during measurements, to avoid desiccation. The natural remanent magnetization (NRM) of the samples was measured at $15 \mathrm{~Hz}$ on a Princeton Applied Research spinner magnetometer. Four pilot samples were then remeasured after each of several demagnetizations in alternating fields up to 200 oersted (Figure 1). The large viscous component was apparently removed by "washing" in about 50 oersted; consequently, all samples were remeasured after 50 oersted af demagnetization.

Table 1 shows the results of NRM and 50 oersted measurements on the Site 212 samples. Inclinations are with respect to the present horizontal, positive downward; negative inclinations indicate normally polarized samples for these southern hemisphere cores. Because the cores were unoriented, declinations are relative only to a given core barrel. Horizontal rotation of sediments within a core due to the drilling process is likely occasionally resulting in a high scatter among declinations from a single core. $A$ and $M M$ are measures of the uncertainty of direction and intensity of magnetization, respectively, based on replicate measurements for each sample. A value of $A$ greater than about 5 or of $M M$ greater than about 25 indicates that the direction or intensity, respectively, of the sample is poorly defined and is thus unreliable.

The NRM's of almost all of the samples are normally polarized (Figure 2). After demagnetization, several samples change to reversed polarity, presumably due to removal of a viscous component parallel to the earth's present field. Even after demagnetization about three-fourths of the samples are normally polarized. If this preponderance of normally polarized samples is due to unremoved viscous components, then the normally polarized samples should have lower inclinations (if the Wharton Basin has experienced a net northward movement) and higher intensities than the reversely polarized samples. Histograms of 50 oersted inclinations (Figure 2) and intensities (Figure 3) show no evidence of significant viscous magnetization.

Cores 15 to 17 are overlain by middle Miocene chalk and underlain by middle Eocene chalk; Cores 27 to 29 are overlain by middle Eocene chalk and underlain by Upper Cretaceous chalk; Cores 36 to 38 are from beneath this Upper Cretaceous chalk. Possibly many of the predominantly normally polarized samples from Cores 27 to 29 and 36 to 38 were deposited during the Upper Cretaceous, a time of dominantly normal polarity (Helsley and Steiner, 1969).

Sclater and Cox (1970) and Sclater and Jarrard (1971) have demonstrated that paleolatitudes calculated from the magnetic inclinations of Deep Sea Drilling cores are consistent with paleolatitudes based on independent lines of evidence. The arrows in Figure 2 show the expected average inclination of the sediments if all of their magnetization was acquired at the present latitude of Site 212 due to an axial geocentric dipole field. The average inclination is considerably steeper than the present dipole field inclination. The possible presence of an offset dipole (Wilson, 1971) or of an unequal dipole pair (Watkins and Richardson, 1973) might account for only $3^{\circ}$ to $4^{\circ}$ of the discrepancy. That compaction affects the inclination seems unlikely (Opdyke and Henry, 1969; Sclater and Jarrard, 1971); its effect, if any, would be to flatten rather than steepen inclinations.

The most likely explanation of the steep inclinations is that the Wharton Basin has moved northward since the deposition of the sediments. Northward motion for the Wharton Basin as well as India and Australia is implied by rapid sea-floor spreading on the Indo-Antarctic Ridge away from a relatively fixed polar Antarctica (Francheteau et al., 1970; Herron, 1972) during the late Tertiary. During its northward movement, the Wharton Basin might be expected to have remained attached to either the Indian or the Australian plate. If the Wharton Basin was attached to the Indian plate, then early Tertiary and Cretaceous paleomagnetic results from India (McDougall and McElhinny, 1970) yield an expected inclination at Site 212 of at least $77^{\circ}$, more likely $80^{\circ}$ to $82^{\circ}$, much steeper than the observed inclinations (Figures 4 and 5).

Figure 6 compares Site 212 paleolatitudes derived from Figure 5 with Australian paleomagnetic data. Virtual geomagnetic poles from Australia (Mumme, 1963; Robertson, 1963, 1966; Robertson and Hastie, 1962; Wellman et al., 1969) were converted to paleolatitudes at Site 212, assuming that the Wharton Basin has remained attached to the Australian plate since the Middle Cretaceous. Because the inclinations in Figure 5 are not normally distributed, a median and confidence limits for the median (Dixon and Massey, 1969, p. 349), rather than a mean and confidence limits for the mean, were calculated from the inclinations of each set of cores of similar age. These median inclinations and their confidence limits $(98 \%$ confidence limits for Cores 15 to 17 and 27 to $29,99 \%$ for Cores 36 to 38 ) were converted to paleolatitudes with the equation $1 / 2 \tan i=\tan$ $p$ and plotted on Figure 6. Cores 15 to 17 and 27 to 29 give 

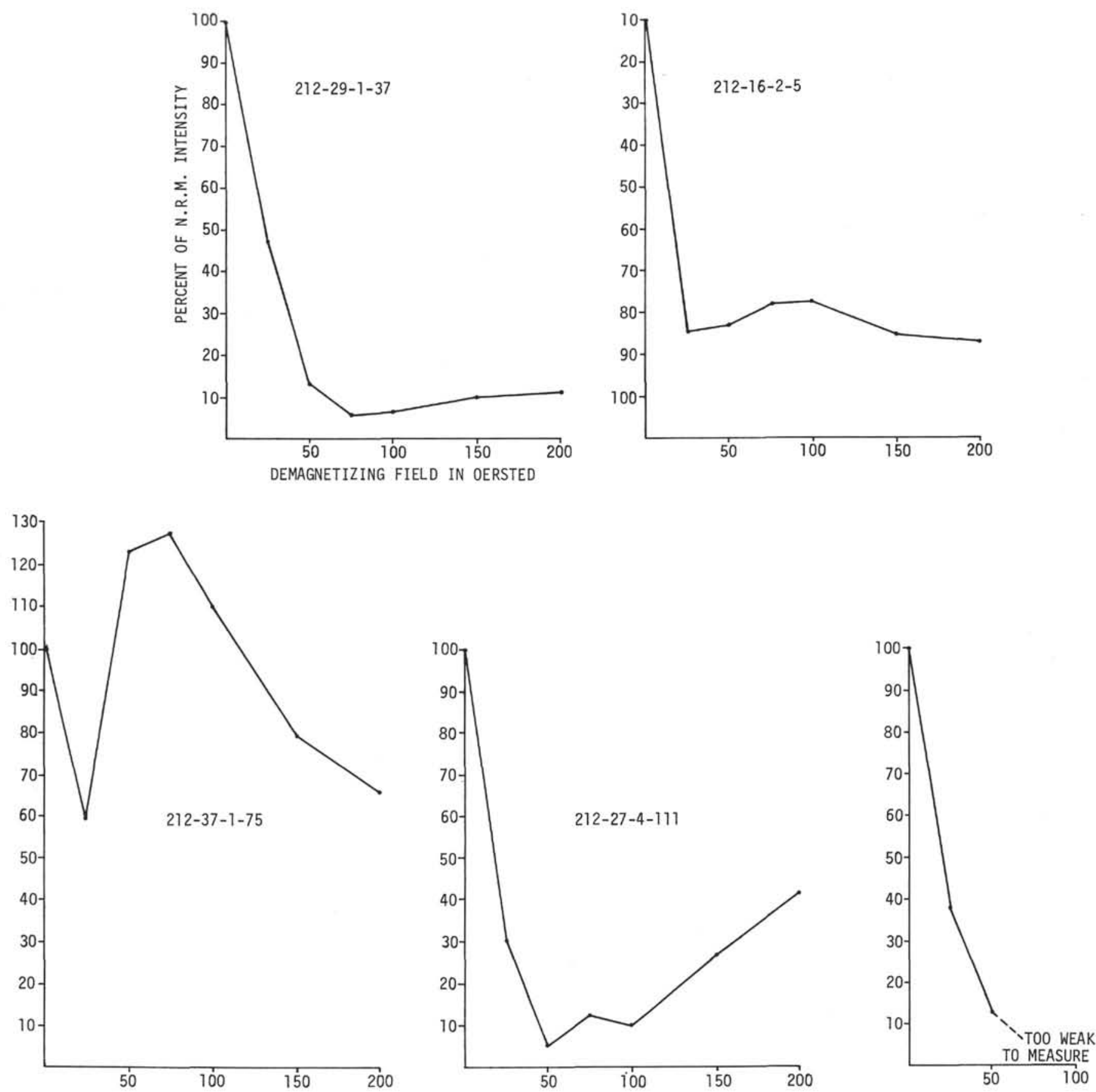

Figure 1. Demagnetization curves of pilot samples.

paleolatitudes significantly different from the present latitude of Site 212. Paleolatitudes from Site 212 sediment cores imply about $5^{\circ}$ to $25^{\circ}$ of northward motion of the site in the late Tertiary and little latitudinal motion in the early Tertiary or late Cretaceous. The paleolatitudes of all three groups of cores are very close to the Australian paleomagnetic results. These paleolatitudes are consistent with the hypothesis that the Wharton Basin has remained attached to the Australian continent since the middle Cretaceous.
An independent line of evidence about movement of the Wharton Basin comes from magnetic anomalies in the basin. As shown by the model studies in Figure 7, the shapes of some magnetic anomalies are quite sensitive to the latitudes at which they were formed. The ANTIPODE XII profile, for example, best matches the model profiles when a paleolatitude of about $25^{\circ} \mathrm{S}$ is assumed at the time of formation of anomalies 27 to 30 and when a paleolatitude of about $30^{\circ} \mathrm{S}$ is assumed for anomalies 30 to $33 \mathrm{~b}$. Models using paleolatitudes more than $10^{\circ}$ from these best-fit 
TABLE 1

Paleomagnetic Measurements of Samples from Site 212

\begin{tabular}{|c|c|c|c|c|c|c|c|c|c|c|c|}
\hline \multirow[b]{2}{*}{ Core-Section } & \multirow{2}{*}{$\begin{array}{l}\text { From Top } \\
\text { of Section } \\
(\mathrm{cm})\end{array}$} & \multicolumn{5}{|c|}{ NRM } & \multicolumn{5}{|c|}{50 Oersted } \\
\hline & & Inclin. & Declin. & $A$ & $\begin{array}{c}\text { Intensity } \\
\times 10^{-5} \mathrm{EMU} / \mathrm{CC} \\
\end{array}$ & $M M$ & Inclin. & Declin. & $A$ & $\begin{array}{c}\text { Intensity } \\
\times 10^{-5} \mathrm{EMU} / \mathrm{CC} \\
\end{array}$ & $M M$ \\
\hline $15-2$ & 95 & -86.4 & 212.3 & 0.0 & 5.3 & 4 & -77.4 & 31.3 & 0.0 & 1.1 & 5 \\
\hline $15-2$ & 97 & -43.2 & 201.7 & 1.8 & 3.0 & 6 & -33.0 & 159.2 & 1.4 & 1.7 & 3 \\
\hline $15-2$ & 99 & -59.2 & 248.8 & 1.5 & 4.7 & 10 & -47.0 & 224.9 & 1.5 & 0.91 & 4 \\
\hline $15-2$ & 101 & -75.4 & 28.5 & 0.2 & 6.2 & 2 & -73.6 & 56.3 & 0.9 & 1.2 & 4 \\
\hline $15-2$ & 103 & 10.0 & 152.2 & 1.1 & 2.4 & 4 & -58.2 & 160.6 & 1.9 & 0.44 & 14 \\
\hline $16-2$ & 1 & -36.2 & 196.1 & 0.7 & 3.5 & 6 & 50.9 & 161.3 & 1.8 & 1.0 & $2 I$ \\
\hline $16-2$ & 3 & -56.4 & 298.4 & 0.1 & 4.4 & 7 & 71.8 & 252.2 & 0.7 & 1.6 & 3 \\
\hline $16-2$ & 5 & -39.7 & 292.5 & 2.6 & 3.1 & 8 & 58.5 & 343.2 & 6.4 & 0.84 & 12 \\
\hline $16-2$ & 7 & -20.7 & 306.9 & 0.4 & 2.6 & 8 & 64.9 & 314.6 & 0.6 & 2.2 & 1 \\
\hline $16-2$ & 123 & -50.3 & 256.5 & 1.2 & 5.9 & 6 & -67.6 & 250.7 & 0.1 & 1.2 & 7 \\
\hline $16-2$ & 127 & -56.5 & 334.3 & 0.9 & 5.6 & 3 & -8.0 & 358.4 & 2.4 & 1.8 & 65 \\
\hline $16-2$ & 129 & -53.7 & 94.6 & 0.1 & 8.2 & 2 & -63.8 & 144.7 & 1.3 & 1.4 & 5 \\
\hline $16-2$ & 131 & -40.3 & 255.4 & 0.2 & 4.5 & 3 & -76.8 & 117.1 & 0.3 & 0.83 & 5 \\
\hline $17-1$ & 100 & -43.6 & 303.2 & 0.0 & 7.2 & 2 & -66.7 & 310.6 & 0.3 & 2.5 & 4 \\
\hline $17-1$ & 102 & -31.2 & 280.3 & 0.9 & 6.9 & 3 & -49.3 & 279.5 & 0.5 & 2.4 & 3 \\
\hline $17-1$ & 104 & -45.8 & 261.1 & 1.0 & 7.8 & 5 & -57.9 & 256.0 & 0.1 & 3.3 & 3 \\
\hline $17-1$ & 106 & -85.1 & 132.0 & 0.1 & 0.89 & 3 & -86.0 & 163.2 & 0.5 & 4.4 & 3 \\
\hline $17-1$ & 136 & -9.1 & 209.2 & 0.0 & 1.3 & 8 & -28.7 & 229.6 & 3.5 & 0.84 & 13 \\
\hline $17-1$ & 138 & -36.2 & 271.2 & 0.5 & 4.7 & 3 & -38.5 & 237.0 & 3.9 & 0.57 & 1 \\
\hline $17-1$ & 140 & -58.0 & 261.5 & 4.1 & 3.0 & 11 & \multicolumn{4}{|c|}{ Too Weak to Measure } & \\
\hline $17-1$ & 142 & -10.1 & 258.6 & 0.3 & 5.8 & 3 & -29.8 & 248.4 & 3.0 & 0.70 & 19 \\
\hline $17-1$ & 144 & -19.4 & 69.7 & 0.1 & 1.8 & 5 & 38.1 & 112.1 & 32.3 & 0.30 & 18 \\
\hline $27-4$ & 3 & 51.7 & 290.7 & 5.0 & 0.58 & 12 & 49.3 & 226.4 & 0.9 & 0.97 & 18 \\
\hline $27-4$ & 5 & -25.4 & 6.2 & 1.3 & 1.2 & 7 & 0.5 & 331.5 & 16.0 & 0.58 & 18 \\
\hline $27-4$ & 109 & $-56: 4$ & 84.6 & 0.2 & 4.3 & 2 & 24.1 & 333.3 & 6.6 & 0.22 & 22 \\
\hline $27-4$ & 111 & -43.8 & 0.2 & 0.1 & 5.5 & 7 & 39.4 & 328.9 & 8.8 & 0.27 & 29 \\
\hline $27-4$ & 113 & -61.2 & 164.4 & 1.7 & 3.7 & 1 & -15.0 & 114.6 & 10.8 & 0.44 & 16 \\
\hline $27-5$ & 63 & -46.2 & 285.2 & 0.0 & 5.4 & 2 & -29.1 & 260.3 & 0.2 & 1.1 & 5 \\
\hline $27-5$ & 65 & -63.0 & 213.8 & 0.9 & 4.6 & 5 & -45.4 & 194.7 & 0.9 & 0.80 & 7 \\
\hline $27-5$ & 67 & -60.9 & 193.8 & 0.2 & 4.6 & 2 & -58.0 & 204.0 & 0.1 & 1.2 & 14 \\
\hline $27-5$ & 69 & -49.7 & 206.6 & 0.3 & 5.0 & 3 & -60.3 & 180.7 & 3.8 & 0.80 & 9 \\
\hline $27-5$ & 118 & -63.8 & 178.5 & 1.5 & 4.4 & 3 & -47.1 & 154.1 & 2.5 & 0.65 & 19 \\
\hline $27-5$ & 120 & -43.6 & 86.4 & 0.5 & 5.3 & 4 & -39.0 & 90.5 & 6.9 & 0.66 & 21 \\
\hline $27-5$ & 122 & -59.5 & 291.6 & 0.2 & 4.7 & 2 & 8.8 & 124.4 & 0.3 & 0.67 & 15 \\
\hline $27-5$ & 124 & -38.8 & 343.3 & 0.5 & 4.9 & 1 & -30.7 & 229.3 & 3.9 & 0.27 & 23 \\
\hline $27-6$ & 20 & 10.9 & 347.3 & 0.0 & 4.4 & 3 & -8.7 & 339.5 & 0.2 & 0.70 & 13 \\
\hline $27-6$ & 22 & -10.5 & 57.8 & 0.3 & 2.9 & 4 & 38.8 & 343.0 & 1.1 & 1.1 & 6 \\
\hline $27-6$ & 24 & 3.0 & 20.3 & 0.1 & 2.6 & 4 & 56.7 & 332.7 & 4.3 & 0.90 & 12 \\
\hline $27-6$ & 26 & 4.9 & 210.2 & 0.2 & 6.5 & 4 & 24.1 & 141.0 & 0.7 & 0.68 & 12 \\
\hline $27-6$ & 28 & -30.7 & 316.3 & 0.1 & 2.6 & 4 & 55.9 & 276.9 & 4.3 & 0.41 & 12 \\
\hline $28-1$ & 0 & -43.6 & 4.5 & 1.4 & 3.8 & 4 & 8.8 & 340.0 & 2.4 & 0.69 & 12 \\
\hline $28-1$ & 2 & -66.1 & 265.0 & 0.0 & 4.5 & 4 & -70.0 & 215.9 & 1.3 & 0.80 & 7 \\
\hline $28-1$ & 4 & -32.2 & 189.3 & 0.0 & 3.9 & 4 & -39.4 & 156.9 & 3.7 & 0.63 & 10 \\
\hline $28-1$ & 6 & -40.5 & 291.8 & 2.1 & 5.7 & 6 & -63.5 & 341.1 & 2.6 & 0.89 & 7 \\
\hline 28-1 & 87 & -85.2 & 332.5 & 0.1 & 5.6 & 4 & -63.9 & 161.1 & 1.3 & 0.80 & 10 \\
\hline $28-1$ & 89 & -65.5 & 348.9 & 0.8 & 6.2 & 4 & -73.6 & 53.9 & 1.5 & 1.0 & 14 \\
\hline 28-1 & 91 & -45.6 & 278.0 & 0.0 & 7.6 & 3 & -40.2 & 280.8 & 0.2 & 0.92 & 10 \\
\hline $28-1$ & 93 & -74.1 & 192.7 & 0.1 & 5.4 & 2 & -54.3 & 101.5 & 2.4 & 0.92 & 18 \\
\hline $28-1$ & 95 & -52.2 & 318.5 & 0.0 & 5.3 & 2 & -44.9 & 310.2 & 2.2 & 0.86 & 11 \\
\hline $28-2$ & 38 & -51.2 & 268.6 & 0.0 & 7.9 & 3 & -51.2 & 237.1 & 1.7 & 1.7 & 3 \\
\hline $28-2$ & 40 & -24.4 & 147.7 & 0.1 & 9.1 & 3 & -59.8 & 118.8 & 0.4 & 2.2 & 6 \\
\hline $28-2$ & 42 & -72.4 & 111.1 & 0.3 & 4.7 & 2 & -50.7 & 130.4 & 2.3 & 1.3 & 6 \\
\hline $28-2$ & 44 & -73.9 & 151.6 & 0.0 & 6.2 & 2 & -11.2 & 127.7 & 0.0 & 0.50 & 15 \\
\hline $28-2$ & 46 & -54.6 & 292.1 & 0.9 & 4.7 & 4 & -40.5 & 287.0 & 0.8 & 0.86 & 10 \\
\hline $28-3$ & 5 & -68.7 & 185.5 & 0.2 & 4.5 & 7 & -54.5 & 184.9 & 3.0 & 1.3 & 9 \\
\hline $28-3$ & 7 & -65.0 & 22.9 & 0.4 & 7.0 & 5 & -43.6 & 59.1 & 1.6 & 1.1 & 8 \\
\hline 28.3 & 9 & -52.0 & 5.7 & 0.6 & 8.0 & 4 & -48.4 & 35.7 & 1.7 & 1.6 & 7 \\
\hline 28.3 & 11 & -43.6 & 5.9 & 0.8 & 10.6 & 1 & -46.5 & 24.6 & 0.9 & 1.4 & 5 \\
\hline $28-3$ & 13 & -53.0 & 324.1 & 0.6 & 7.9 & 2 & -67.3 & 0.1 & 0.2 & 1.4 & 8 \\
\hline $28-3$ & 15 & -66.8 & 264.0 & 0.0 & 5.2 & 4 & & & & & \\
\hline $28-3$ & 17 & -34.6 & 307.5 & 0.4 & 4.0 & 10 & & & & & \\
\hline
\end{tabular}


TABLE 1 - Continued

\begin{tabular}{|c|c|c|c|c|c|c|c|c|c|c|c|}
\hline \multirow[b]{2}{*}{ Core-Section } & \multirow{2}{*}{$\begin{array}{l}\text { From Top } \\
\text { of Section } \\
(\mathrm{cm})\end{array}$} & \multicolumn{5}{|c|}{ NRM } & \multicolumn{5}{|c|}{50 Oersted } \\
\hline & & Inclin. & Declin. & $A$ & $\begin{array}{c}\text { Intensity } \\
\times 10^{-5} \mathrm{EMU} / \mathrm{CC} \\
\end{array}$ & $M M$ & Inclin. & Declin. & $A$ & $\begin{array}{c}\text { Intensity } \\
\times 10^{-5} \mathrm{EMU} / \mathrm{CC} \\
\end{array}$ & $M M$ \\
\hline $29-1$ & 35 & -57.4 & 329.7 & 0.9 & 4.3 & 4 & -53.4 & 315.6 & 1.4 & 0.58 & 19 \\
\hline $29-1$ & 37 & -53.6 & 5.1 & 0.3 & 5.4 & 2 & -39.0 & 359.2 & 10.7 & 0.73 & 17 \\
\hline $29-1$ & 39 & -82.4 & 143.4 & 0.4 & 4.5 & 3 & -66.9 & 56.7 & 1.3 & 0.60 & 12 \\
\hline $29-1$ & 41 & -64.6 & 84.1 & 1.0 & 4.6 & 5 & -52.0 & 53.6 & 3.5 & 1.1 & 6 \\
\hline $29-1$ & 117 & -40.5 & 25.2 & 0.1 & 5.0 & 3 & -33.2 & 5.7 & 1.7 & 1.6 & 4 \\
\hline $29-1$ & 119 & -58.2 & 284.2 & 0.5 & 6.1 & 1 & -49.1 & 244.0 & 0.5 & 2.2 & 3 \\
\hline $29-1$ & 121 & -59.9 & 83.1 & 0.0 & 7.0 & 2 & -38.5 & 76.9 & 1.5 & 1.9 & 9 \\
\hline $29-1$ & 123 & -48.9 & 143.7 & 1.6 & 8.5 & 1 & -35.0 & 124.2 & 0.3 & 2.6 & 4 \\
\hline $36-1$ & 15 & -66.8 & 264.0 & 0.0 & 5.2 & 4 & \multicolumn{5}{|c|}{ Too Weak to Measure } \\
\hline $36-1$ & 17 & -34.6 & 307.5 & 0.4 & 4.0 & 10 & -19.7 & 263.4 & 1.8 & 0.42 & 18 \\
\hline $36-1$ & 19 & -50.4 & 320.3 & 1.2 & 6.9 & 5 & -33.7 & 261.7 & 0.1 & 1.7 & 2 \\
\hline $37-1$ & 73 & 5.2 & 8.6 & 0.4 & 2.8 & 5 & 56.8 & 252.9 & 1.2 & 3.1 & 4 \\
\hline $37-1$ & 75 & -19.8 & 357.0 & 0.1 & 2.4 & 4 & 58.1 & 185.3 & 1.2 & 3.0 & 3 \\
\hline $37-1$ & 111 & -58.1 & 306.7 & 0.6 & 7.7 & 2 & -58.5 & 287.6 & 1.1 & 3.2 & 2 \\
\hline $37-1$ & 113 & -77.5 & 256.7 & 0.2 & 6.2 & 4 & -62.9 & 198.5 & 0.3 & 3.0 & 2 \\
\hline $37-1$ & 115 & -51.3 & 307.9 & 0.5 & 7.1 & 4 & -66.6 & 262.0 & 0.2 & 2.5 & 3 \\
\hline $38-2$ & 69 & -51.3 & 76.8 & 0.6 & 8.7 & 3 & 79.7 & 119.3 & 8.3 & 4.2 & 58 \\
\hline $38-2$ & 71 & -34.5 & 358.6 & 0.0 & 12.8 & 2 & -4.9 & 38.5 & 0.0 & 8.2 & 1 \\
\hline $38-2$ & 73 & -27.9 & 249.2 & 0.7 & 4.5 & 4 & -24.5 & 259.8 & 0.5 & 2.7 & 5 \\
\hline $38-2$ & 75 & -34.3 & 270.7 & 0.6 & 7.2 & 3 & -26.0 & 235.5 & 1.7 & 1.7 & 7 \\
\hline $38-3$ & 13 & & & & & & -67.3 & 0.1 & 0.2 & 1.4 & 8 \\
\hline
\end{tabular}
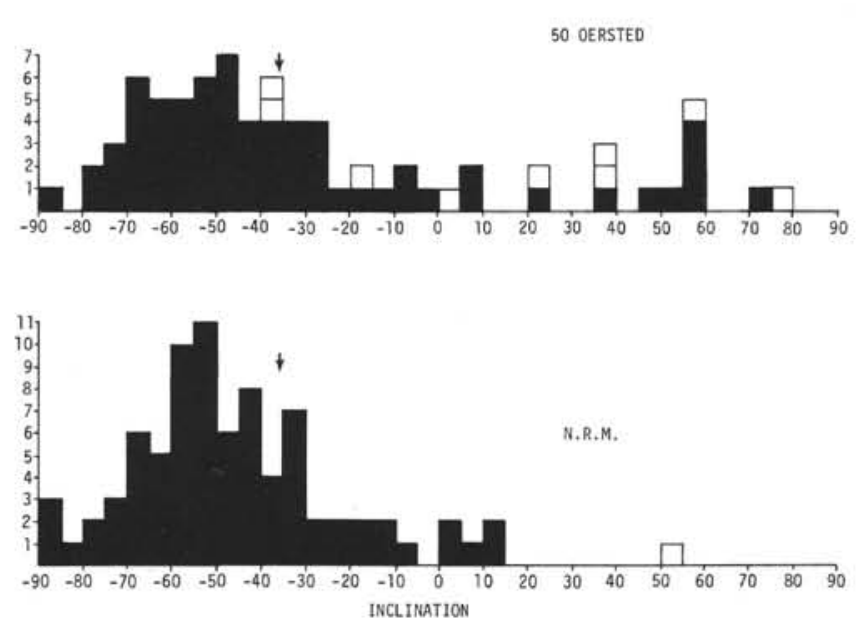

Figure 2. Histograms of all sample inclinations before and after 50 oersted demagnetization. Shaded squares indicate reliable inclination measurements; unshaded squares indicate unreliable $(\mathrm{A} \geqslant 5.0)$ measurements.

paleolatitudes show a considerably lower quality fit. These paleolatitudes are significantly different from the present latitudes of the anomalies and very close to expected paleolatitudes (Figures 8 and 9) based on Australian paleomagnetic data, assuming that the Wharton Basin has remained attached to Australia.

\section{REFERENCES}

Dixon, W. J. and Massey, F. J., Jr., 1969. Introduction to Statistical Analysis: New York (McGraw Hill).
Francheteau, J., Harrison, C. G. A., Sclater, J. G., and Richards, M.L., 1970. Magnetization of Pacific seamounts: a preliminary polar curve for the northeastern Pacific: J. Geophys. Res., v. 75, p. 2035-2061.

Helsley, C. E. and Steiner, M. B., 1969. Evidence for long intervals of normal polarity during the Cretaceous period: Earth Planet. Sci. Lett., v. 5, p. 325-332.

Herron, E. M., 1972. Sea-floor spreading and the Cenozoic history of the east-central Pacific: Geol. Soc. Am. Bull., v. 83 , p. $1671-1692$.

McDougall, I. and McElhinny, M. W., 1970. The Rajmahal Traps of India-K-Ar ages and paleomagnetism: Earth Planet. Sci. Lett., v. 9, p. 371-378.

Mumme, W. G., 1963. Thermal and alternating field demagnetization studies on Cenozoic basalts of Victoria, Australia: Geophys. J. Roy. Astr. Soc., v. 7, p. 314.

Opdyke, N. D. and Henry, K. W., 1969. A test of the dipole hypothesis: Earth Planet. Sci. Lett., v. 6, p. 139-151.

Robertson, W. A., 1963. The paleomagnetism of some Mesozoic intrusives and tuffs from eastern Australia: J. Geophys. Res., v. 68, p. 2299-2312. 1966. Paleomagnetism of some Cenozoic igneous rocks from south-east Queensland: Proc. Roy. Soc. Qd., p. 78-87.

Robertson, W. A. and Hastie, L., 1962. A paleomagnetic study of the Cynet alkaline complex of Tasmania: J. Geol. Soc. Australia, v. 8, 259-268.

Sclater, J. G. and Cox, A., 1970. Paleolatitudes from JOIDES deep sea sediment cores: Nature, v. 226, p. 934-935.

Sclater, J. G. and Jarrard, R. D., 1971. Preliminary paleomagnetic results, Leg 7. In Winterer et al., Initial Reports of the Deep Sea Drilling Project, Volume VII: Washington (U.S. Government Printing Office), p. 1227-1234. 

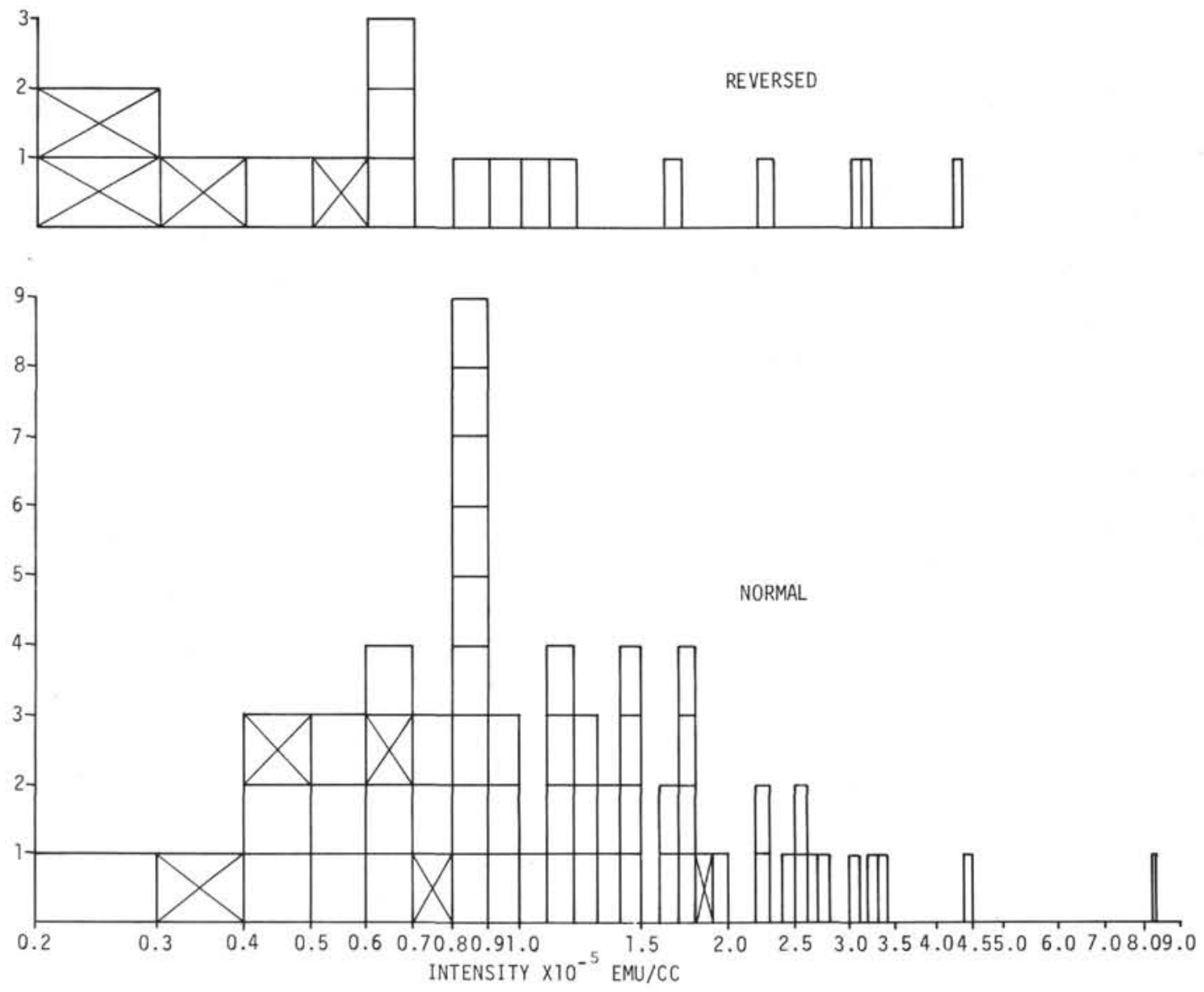

N.R.M.

REVERSED

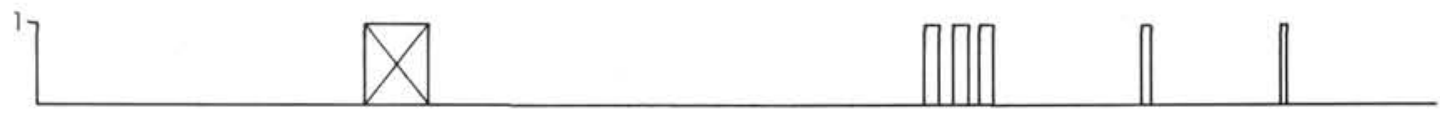

NORMAL

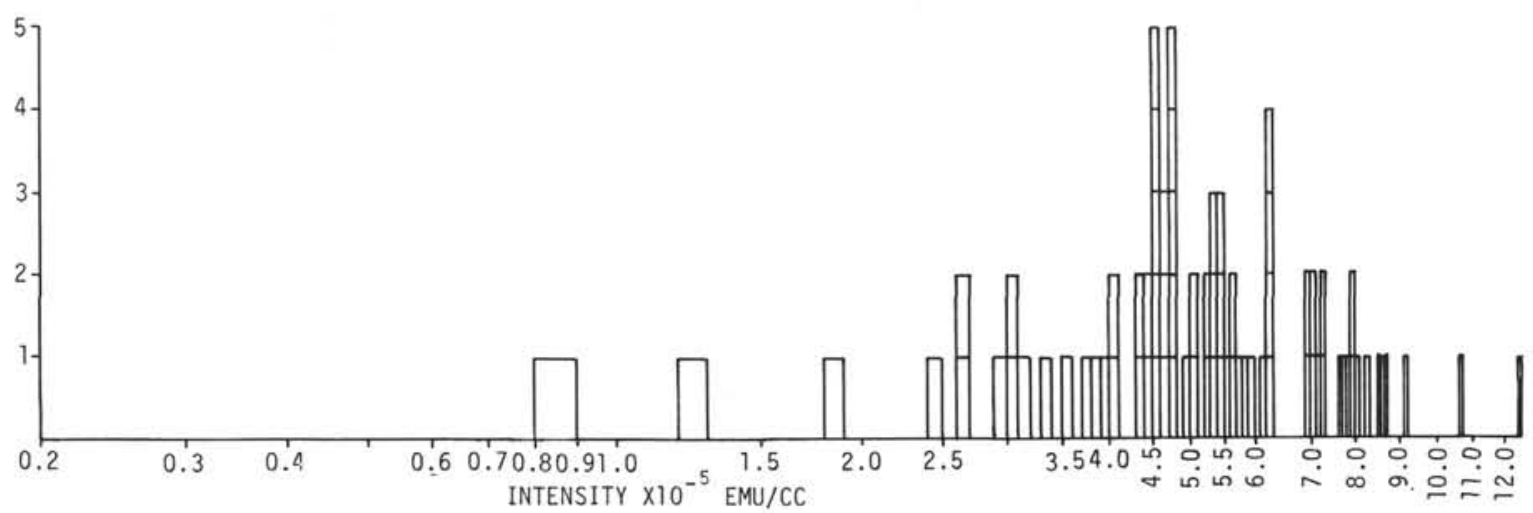

Figure 3. Histograms of all intensities for normally and reversed polarity samples before and after 50 oersted demagnetization. Horizontal scale is logarithmic. Each rectangle represents one sample. Rectangles containing an $X$ are intensities from unreliable $(\mathrm{A} \geqslant 5.0)$ measurements. 
Watkins, N. D. and Richardson, A., 1973. Analysis of upper Tertiary paleomagnetic data in terms of an unequal co-axial dipole pair geomagnetic field model: EOS (Trans. Am. Geophys. Union), v. 54, p. 253.

Wellman, P., McElhinny, M. W., and McDougall, I., 1969. On the polar wandering path for Australia during the Cenozoic: Geophys. J. Roy. Astr. Soc., v. 18, p. 371-395.

Wilson, R. L., 1971. Dipole offset-the time average paleomagnetic field over the past 25 million years: Geophys. J. Roy. Astr. Soc., v. 22, p. 491-504.

\section{CORES $15-17$}

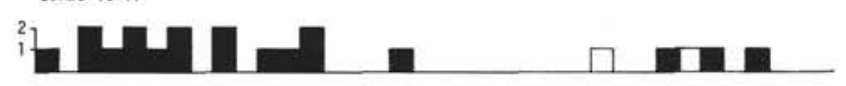

CORES $27-29$
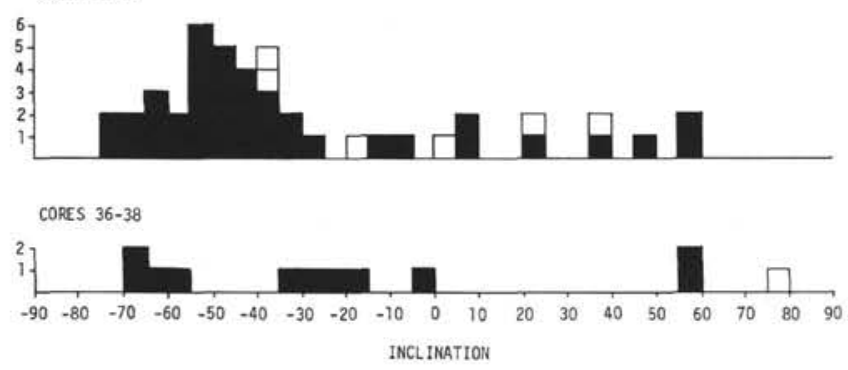

Figure 4. Histograms of sample inclinations after 50 oersted demagnetization. Shaded squares indicate reliable inclination measurements; unshaded squares indicate unreliable $(\mathrm{A} \geqslant 5.0)$ measurements.

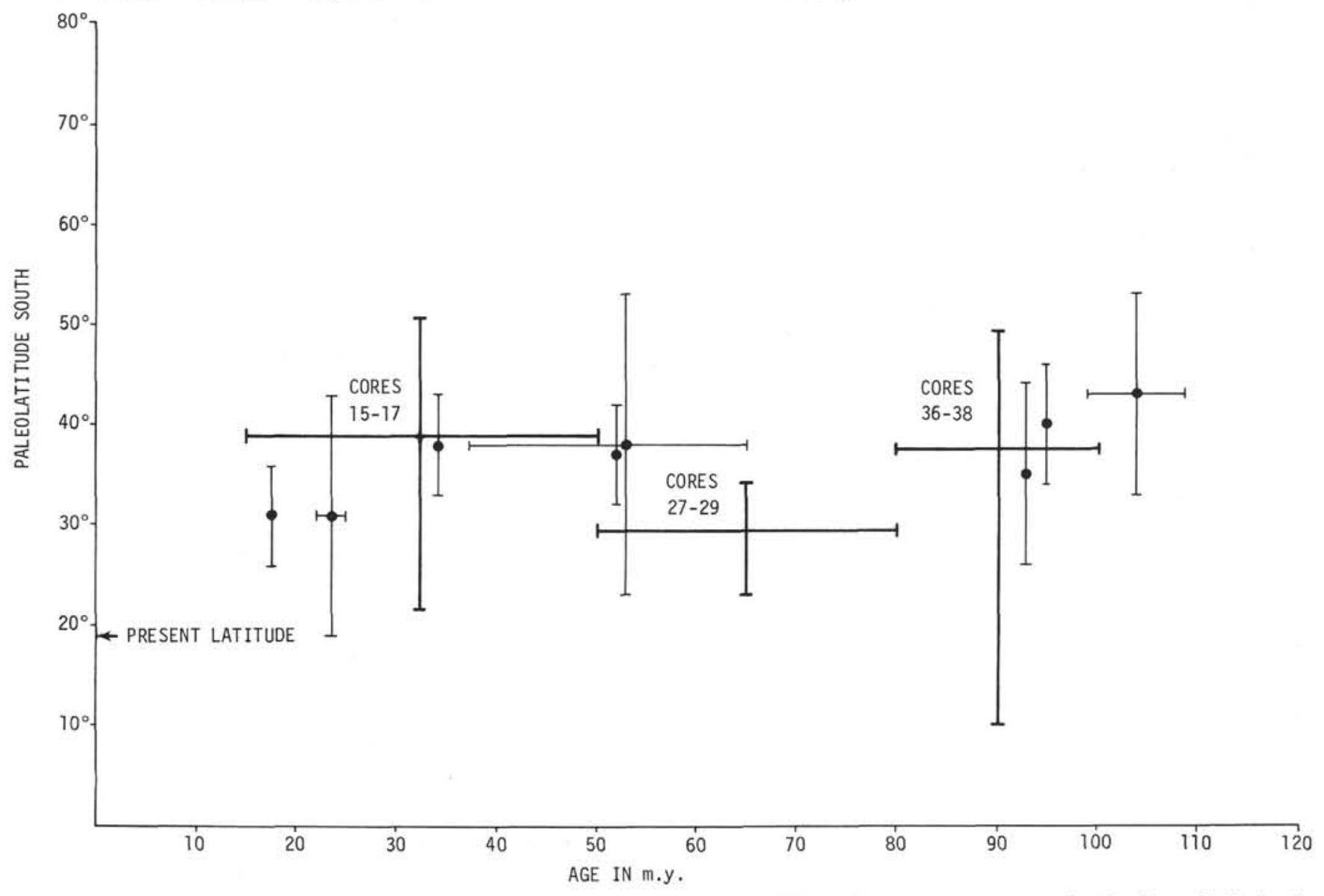

Figure 6. Plot of paleolatitude information for Site 212 through time. Heavy bars represent error limits for paleolatitudes calculated from inclination data shown in Figure 5. Solid dots and lines indicate paleolatitudes and their error bars derived from Australian paleomagnetic poles.
CORES 15-17

CORES 27-29

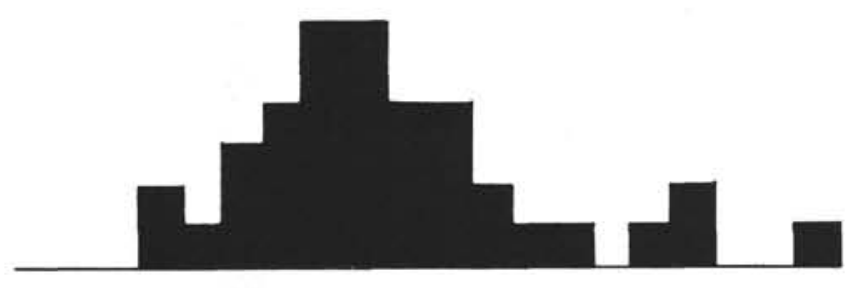

CORES $36-38$

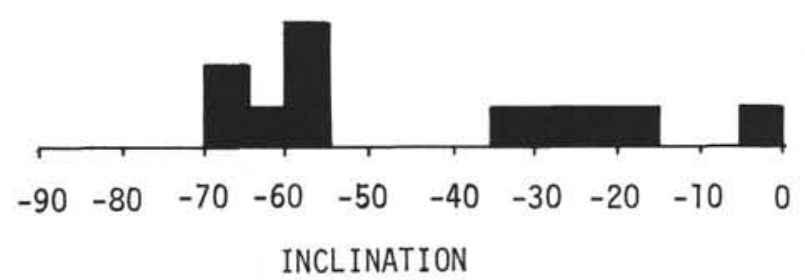

Figure 5. Histograms of reliable sample inclinations after 50 oersted demagnetization. Reversely magnetized samples have been changed to normal polarity (negative inclination). 

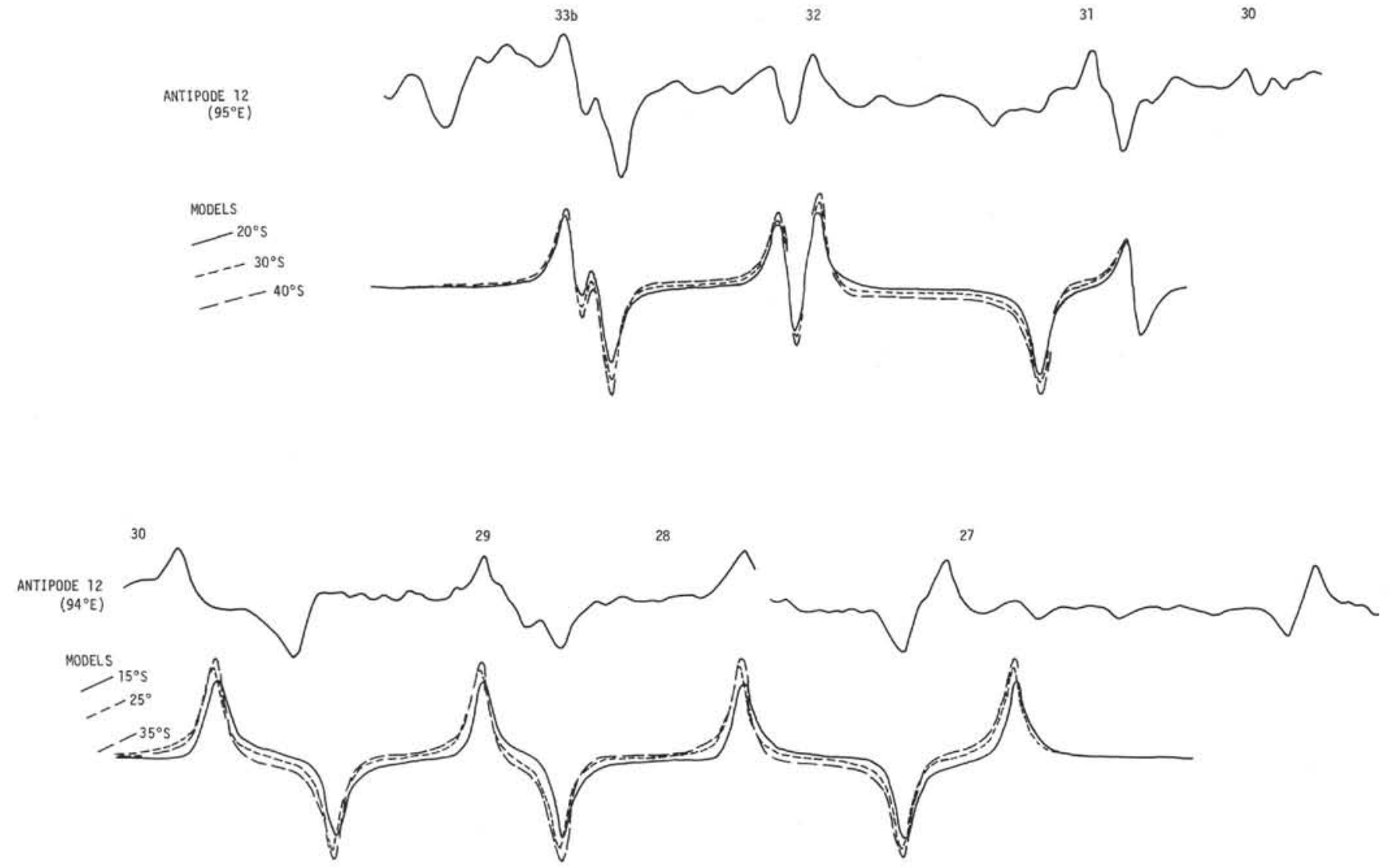

Figure 7. Example of magnetic anomaly profiles in the Wharton Basin compared to models assuming various latitudes of crustal origin.

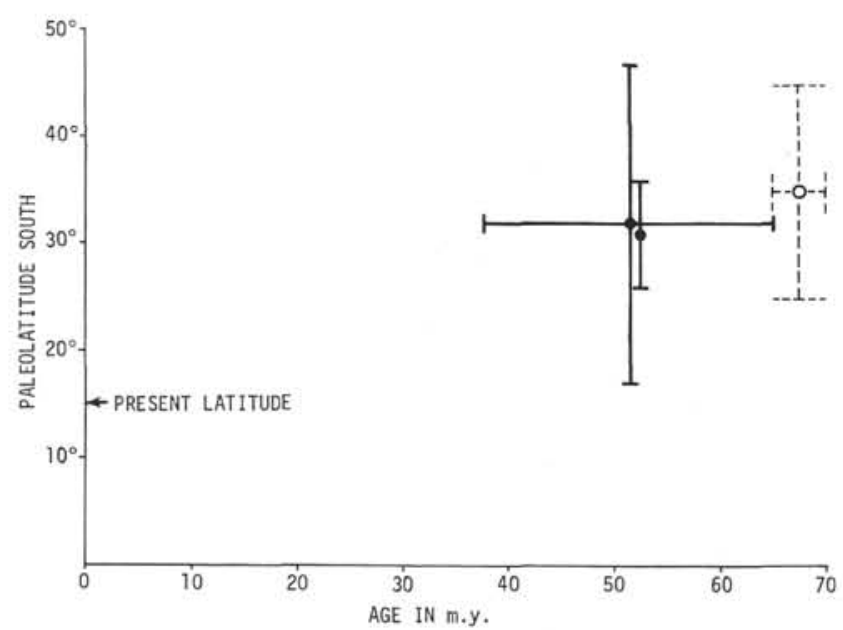

Figure 8. Comparison of paleolatitude from magnetic anomalies 30 to $33 \mathrm{~b}$ on ANTIPODE XII profile with paleolatitudes derived from Australian VGP's by assuming that the Wharton Basin has remained attached to Australia.

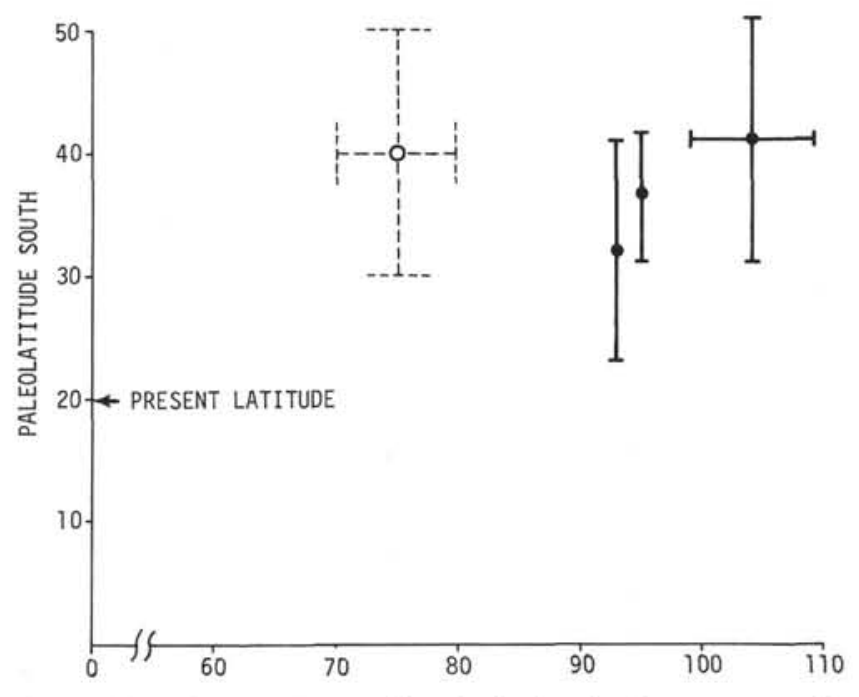

Figure 9. Comparison of paleolatitude from magnetic anomalies 27 to 30 on ANTIPODE XII profiles with paleolatitudes derived from Australian VGP's by assuming that the Wharton Basin has remained attached to Australia. 